\title{
A Bacillus subtilis gene cluster similar to the Escherichia coli phosphate-specific transport (pst) operon: evidence for a tandemly arranged pst $B$ gene
}

\author{
Ken-ichi Takemaru, $†$ Motoki Mizuno and Yasuo Kobayashi \\ Author for correspondence: Yasuo Kobavashi. Tel: +81423675706 . Fax: +81423675715 . \\ e-mail:ykobayas@cc.tuat.ac.jp
}

Faculty of Agriculture,

Tokyo University of

Agriculture and

Technology, Fuchu, Tokyo

183, Japan

\begin{abstract}
We have determined the complete nucleotide sequence of the Bacillus subtilis homologues of the Escherichia coli phosphate-specific transport (pst) genes in the framework of the international $B$. subtilis genome sequencing project. The pst genes in $E$. coli form an operon arranged in the order pstS, pstC, pstA, pstB and phoU. In the case of $B$. subtilis, there are also five ORFs presumably forming an operon. The deduced amino acid sequences of the products of these ORFs show striking similarities to their $E$. coli counterparts. Comparison of the organization of the pst operon of B. subtilis with that of $E$. coli revealed that the gene corresponding to phoU is missing, while there are two genes homologous to pst $B$ in $B$. subtilis. The pst operon is located at $222^{\circ}$ on the $B$. subtilis chromosome.
\end{abstract}

Keywords: Bacillus subtilis, genome sequencing project, phosphate-specific transport operon, pst $B$, tandem genes
The phosphate-specific transport (Pst) system of Escherichia coli comprises four distinct subunits encoded by the pst $S$, pst $A$, pst $B$ and pst $C$ genes (Amemura et al., 1985; Surin et al., 1985). These genes, together with the phoU gene, form the pst operon, which maps at about 84 min on the E. coli chromosome (Bachmann, 1990). The nucleotide sequences of all five genes have been determined, and the amino acid sequences of the corresponding proteins have been deduced (Amemura $e t$ al., 1985; Surin et al., 1985). Apart from transporting phosphate, the Pst system plays an important role in the regulation of a number of coordinately regulated genes collectively referred to as the phosphate regulon (Wanner, 1987; Wanner \& Letterel, 1980).

As part of the international Bacillus subtilis sequencing project, we report here the cloning and sequencing of $B$. subtilis homologues of the E. coli pst gene products.

A $\lambda$ phage library of B. subtilis strain $\mathrm{JH} 642(\operatorname{trp} C 2$ phe A1)

\footnotetext{
†Present address: Department of Genetics, The Graduate University for Advanced Studies, 1111 Yata, Mishima, Shizuoka-ken 411, Japan.

Abbreviations: Pst, phosphate-specific transport; RBS, ribosome-binding site.

The DDBJ accession number for the nucleotide sequence reported in this paper is $D 58414$.
}

chromosomal DNA was constructed as described previously (Takemaru et al., 1995). The library was screened using as a probe plasmid pNEXT27, which is one of the Not I linking clones containing a DNA insert located at $222^{\circ}$ on the B. subtilis chromosome (Itaya \& Tanaka, 1991). With this probe, several recombinant $\lambda$ phage clones covering the entire region of the $B$. subtilis pst operon were isolated. Sequencing was performed by a strategy based on the combination of PCR and the random sequencing method, i.e. preparation of a random library, amplification of inserts subcloned in M13 phages by PCR, sequencing of randomly selected inserts, and filling of the gaps by region-specific primers (Ogasawara et al., 1994). The entire DNA sequence was determined on both strands to ensure high accuracy.

The complete nucleotide sequence of $B$. subtilis pst operon is shown in Fig. 1 with the deduced amino acid sequences of five ORFs. These ORFs are transcribed in the same direction, and each is preceded by a putative ribosomebinding site (RBS) appropriately spaced from a presumed ATG initiation codon. The canonical vegetative promoter sequence is found upstream of ORF108. A perfect $15 \mathrm{bp}$ inverted repeat that is a candidate for a transcriptional termination site is found downstream of ORF75. In addition, two sequences showing similarities with $E$. coli pho boxes are observed in the upstream region of ORF108. 


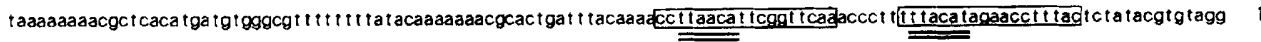
ORF108 start

acaaa $t$ t acacat t a t acgcagggggaa t tcaggaaATGAAAAAAAACAAATTGGTGCTTATGCTTCTGATGGCTGCTITTATGATGATTGCAGCTGCATGCGGAATGCAGGAGAAAGT 240 M K K N K L V L M L L M A A F M I I A A A C G N A G E S 28

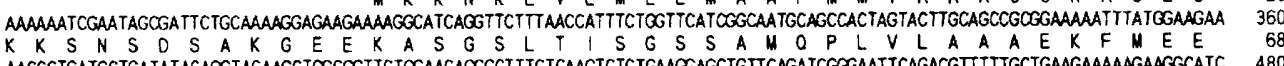
AACCCTGATGCTGATATACAGG TACAAGCTGGCGGTTCTGGAACAGGGCTTTCTCAAGTCTCTGAAGGAGCTGTTCAGATCGGGATT TCAGAGGT TTGGCTGAGGAALAGGAGGCATC $N$ P D A D I O V O A G G S G T G L S O $V$ S E G A V O I G

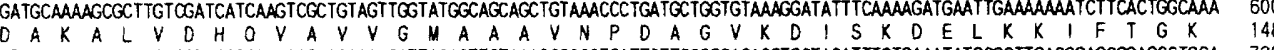
ATCAAAAACTGGAAAGAGCTCGGCGGCAAAGACCAAAAGATTACACT TGTAAACOGCCCT TGATTCTTCCCGCACACGTGCTACATTTGTGAAATATGCGCTTGACGGAGCGGAGCCTGCA 720 $K$
$I$

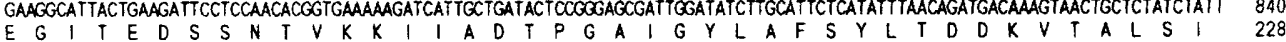
GACGGTGTTAAACCTGAAGCGAAAAATGTAGCAACTGGCGAGTATCCGATCTGGGCTTATCAACATTCTTATACAAAGGGGGAGGCGACAGGTTTAGCTAAAGAATTTCTTGATTATCTA 960

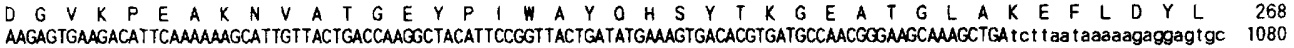

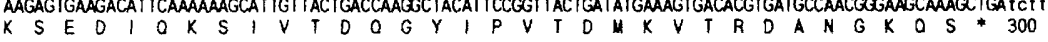

ORF72 start

ggggacaaccgt t tctcc tct taacacat tcataggagt gt aaaacaATGATAAACAATAGAGAAAATATGTCTGTGAGOGAGCGCTTAATCAGCTCTAGGCAAAATOGGCAATGGATG 1200 M I N N A E N M S V S E R L I S S R O N R O L D E 25 AAGTTCGGGGAAGAATGATAGTCACAGCTTGTGCACTGATTATGATTGOGGCATCTGTTGCGATTACCATTTTCCTOGGOGTTAAOGGGCTGCAATCTTTTCTTGTAAATGGGTAAGCC 1320

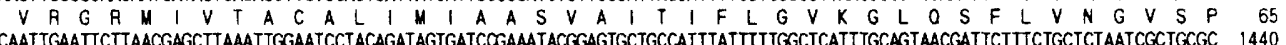
CAATTGAATTCTTAACGAGCTTAAATTGGAATCCTACAGATAGTGATCCGAAATACGGAGTGCTGCCATTTATTTITGGCTCATTTGCAGTAACGATTCTTTCTGCTCTAATCGCTGCGC 1440

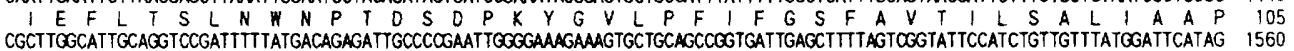
$L G$ I A G P I F M T E I A P N W G K K V L O P V I E L L V G I P S V V Y G F I G 145 GTCTTACAGTATTGGTGCCGTTTATCGCCCAGTTCAAATOGAGCGGAACAGGGCACAGTTTATTGGOOOGAACGATTGTCCTTTCOGTCATGATTCTGCCGACGATCACATCGATTTCTG 1680 L T V L L V F P F F I A A O F K K S S S G T G G H S S L L A G T I V L L S V V M I L L P T I T S I S A 185 D A M A S L P K S L A E G S Y A L G A T R O T I R K V L V P A A F P T L M T A 225 CTGTTGTGITAGGCATGCCTCGGGCGICGGTGAGGTTIGGCTGITCAGATGGTCATTGGAAATACAAGAGTATTOCCOGAAAGCCCGITTGACACTGCGGGAACGCTGACAACGATCA 1920

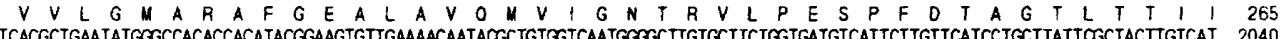
TCACGCTGAATATGGGCCACACCACATACGGAAGTGTIGAAAACAATACGCTGTGGTCAATGCOGCTIGTGCTTCTGGTGATGTCATTCTTGITCATCCTGCTTATTOGCTACTTGTCAT 2040 ORF73 start CTAGGAGGAAAGTGTAATGAACCGCAAAATAACAGATAAACTGGCGACOGGAATGTTTGGTTTGTGTGCAGCCATTATCOCTGCCATTTIAGTAGGTTTGTTTTCCTATATTATTATAAA 2160

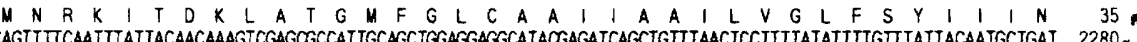
CGGTGTTTCTCAGCTCAGTTTCAATTATTACACAMAGTCAGCGCCATTGCAGCGGGOGGGCATACOGATCGGTG

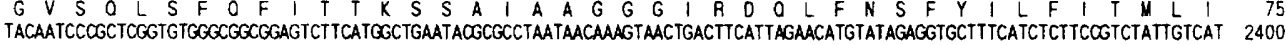
\begin{tabular}{l|lllllll} 
&
\end{tabular} TGGGATGTTGGATIATTGATGTIGTCAACTIAACAGGCTGCGGTATACGATCATAGGCGGAGCGCTTGCGTTACTGTITITACCTTCCTGTCATGGTGCGTGTGACAGAAGACGC 2520 $G M F G L L M F V N L T G W G$ M I I I G G A L A L T V F N L P V M V B V T E D A GATCCG TCAGTCCCTAAAGATCTGAAGGAAGCCTCTCTCGCTTTAGGTGTATCACGCTGGCATACOGTAAAAACAGTTTIAATTCCAAGTGCGATTCCTTCTATCATCACAGGAGCAAT 2640 I R S V P K D L K E A S L A L G V S R H H T Y K I V L I P S A I P S I I I G A I 195 TTTAGCGTCAGGAaGGG TGTTGGGGAaGCGGCOGCATTATTATTTACGGCAGGCCTGACAACACCGCGTCTTAATT TACGGAGTGGAACCCGTTTCGGAaCATCACCGCTTAATAT 2760 TTTCAGGCCCGCTGAAACACTIGOGGTCCACATTTGCAACGTCAATACACAAGGAATGATTCCGGATGCTGAAGCGATTGCCAACGGTOGTICTCCCGTGCTGGTGATTICTGTTCTTGT 2880 F A P A E T L V H W W V N T O G I P D A E A I A N G G S P V L VIS V L V 275

OFF74 start

GTTTAATCTCGCGGGGAGATGGCTTGGCACGATGATCTACAAAAAGCTTACGGCAAATTAGaacggaaagaggogaaaaacATGICTGAACAAATGGTAAAAGAAAAACCTGAGCGAGCT 3000 F N L A A R W L G T I I Y K K L T A N * 294 U S E O WVK K K P E R A 13 GTTATTGTTCCGAAGCAGAACCACGTGCTTGAGGTCAAAGATCTGTCCATTTATTACGGAAATAAACAAGCCGTTCATCATGTAAACATGGATATTGAAAAAAATGCGGTGACTGCTTTA 3120 \begin{tabular}{llllllllllllllllllllllllllllllllllllllll}
$V$ & $I$ & $V$ & $P$ & $K$ & $O$ & $N$ & $H$ & $V$ & $L$ & $E$ & $V$ & $K$ & $D$ & $L$ & $S$ & $I$ & $Y$ & $Y$ & $G$ & $N$ & $K$ & $O$ & $A$ & $V$ & $H$ & $H$ & $V$ & $N$ & $M$ & $D$ & $I$ & $E$ & $K$ & $N$ & $A$ & $V$ & $T$ & $A$ & $L$ \\
\hline
\end{tabular} ATTGGCCGTCGGGTGCGGAAAATCTACTTTTITGAGAAATATTAACOGAATGAACGATTTAATTCCATCTGCAAGAGCGGAAGGTGAAATCCTTTATGAGGGATTAAATATATTGGG 3240

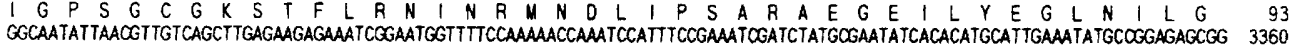

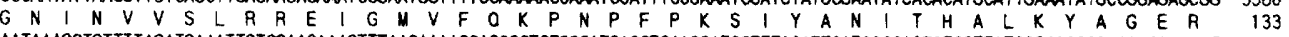
AATAAAGCTGTTT TAGATGAAATTGTGGAGAAAGTTTAACAAAGCAGCGCTCTGOGATGAGGTGAAGGATCGTTTACATTCATCCGCACTCTCGTTATCCGGCOGCCAGCAGCAGCGT 3480 TTATGCATOGCGAGAACGCTTGCGATGAAGCCGOC TGTTCTTCTGCTTGATGAACCAGCTTCAGCGCTTGATCCGATTICAAATGCAAAAATAGAGGAATTAATTACAGGATTGAAAAGA 3600 $L$ C I A R T L A N K P A V L L L D E P A S A L D P I S N A K I E E L I T G L K R 213 GAATACTCAATTATTAT TGTCACGCACAATATGCAGCAGGCTCTGCGGGTTTCTGACOGGACGGCATTCTTTTTAAACGGCGAGCTTGTAGAATACGGACAGACCGAACAGATTTTCACG 3720 E Y S I I I V T H N M O O A L R V S D R T A F F L N GEL VE Y G O T E O I F T 253

\section{ORF75 start}

AGTCCGAAAAGCAAAAGACAGAGGATTATATTAACGGGAAATTCGATAGgaggocggcaATGAGTATTGCTACCGAAGCTGTAATGAAACAGGAGTATATCAAGTCAATGGAATGAA 3840

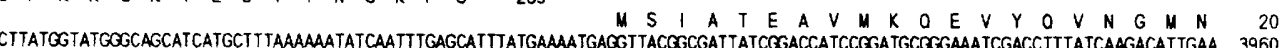

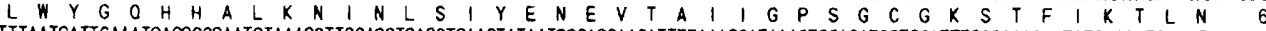
TTTAATGATTCAAATGAOGCCGAATGTAAAGCTTGCAGGTGAGCTGAACTATAATGGCAGCAACATTTTAAAGGATAAAGTGGACATCGTGGATTTGCGAAAAAATATCGGCATGGTATT 4080

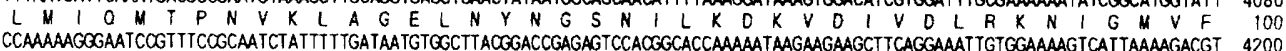

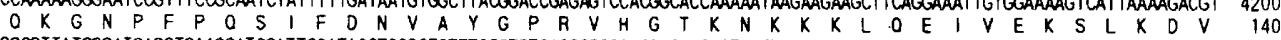
GGCGTTATGGGATGAGGTGAAGGATCGATTGCATACGTCCGCICTITCGCTGTCAGGOGGCCAGCAGCAGCGICTTTGCATCGCCAGAGCACTIGCGACCAATCCTGATATITTGCTGAT 1432 $A L L W D E V V K$ D R L H T S A L S L S G G O O O O R L C I A R A L A T N P D I L L M GGATGAACCGACAICTGCACTAGATCCAATCTCAACGAGAAAAATTGAAGAACTCATICTAGAGCTGAAAGATAAATATACAATIGTCAT TGTCACGCATAACATGCAGCAGGCAGCAAG 4440

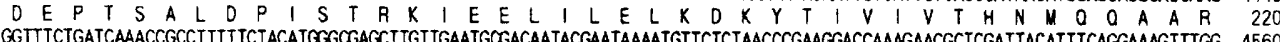

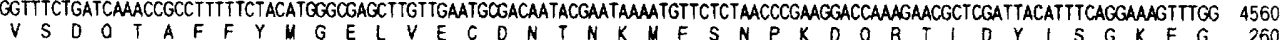

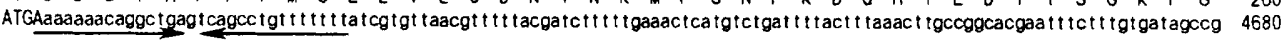
* 260

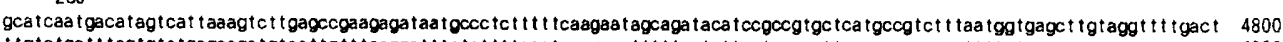

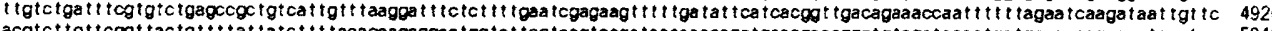
acgtct tgt tcgot tac tgit t t at tatct t t tacagcagcggcc ggtct tcglcagtcaga taaaaacagct gcaagaacggctgtcgct aaaa tga tgccgccagcaaa tgcct ga 5040

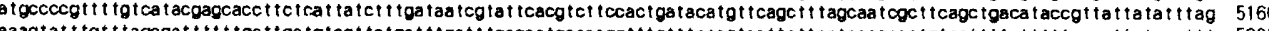

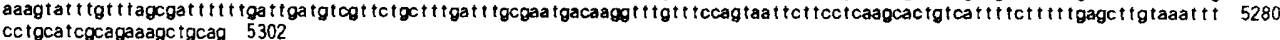

Fig. 1. For legend see facing page. 


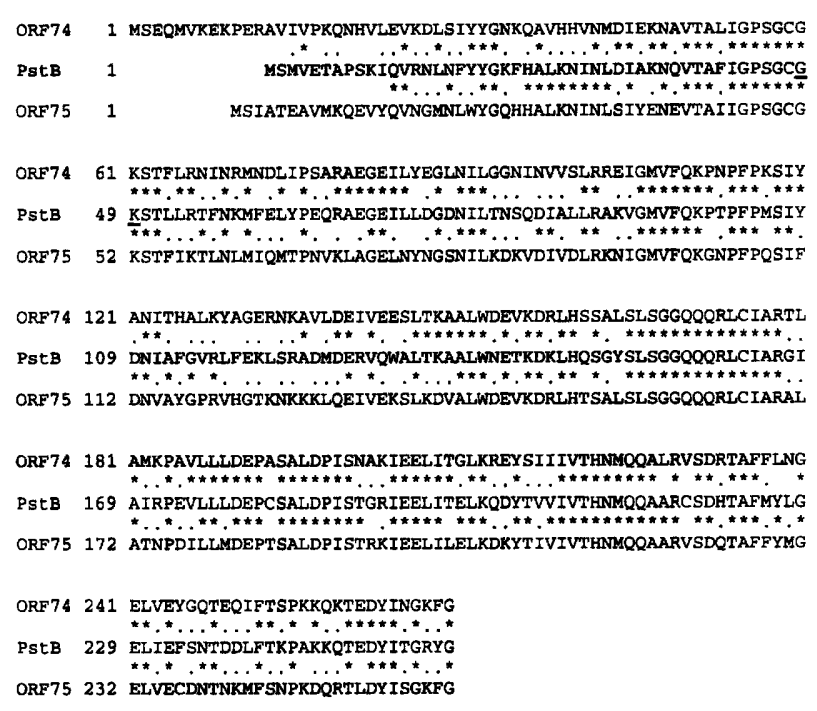

Fig. 2. Alignments of the amino acid sequences of the predicted products of ORFs 74 and 75 with $E$. coli PstB. The two key residues (Gly-48 and Lys-49) of the E. coli PstB protein, which have been shown to be important for phosphate transport (Cox et al., 1989), are underlined. Hyphens indicate gaps introduced to improve the alignments. Asterisks and dots represent identical and conserved amino acid residues, respectively. The amino acid sequence of $E$. coli PstB is from Amemura et al. (1985) and Surin et al. (1985).

It was demonstrated that $E$. coli $\mathrm{PhoB}$ protein, a response regulator of the bacterial two-component regulatory system, bound to the pho box and activated the transcription of pst operon in vitro (Makino et al., 1988). We believe that the $B$. subtilis pho box-binding protein will recognize these sequences and regulate the transcription of this operon.

The deduced amino acid sequence of ORF108 shows 20.7\% identity to the PstS protein, which is a phosphatebinding protein located in the periplasmic space (Gerdes \& Rosenberg, 1974). The ORF72 product has $26.1 \%$ identity to the PstC protein, and the protein encoded by ORF73 has $27 \cdot 2 \%$ identity with the PstA protein. The PstA and PstC proteins are hydrophobic and likely to form the transmembrane portion of the Pst system (Amemura et al., 1985; Surin et al., 1985). The hydropathy plots of the ORF72 and ORF73 products show significant similarities to those of their E. coli counterparts (data not shown). Interestingly, the ORF74 and ORF75 products show $58.6 \%$ identity with each other and $57 \cdot 3 \%$ identity to the PstB protein (Fig. 2). The PstB protein is hydrophilic and is likely to interact on the cytoplasmic side with the PstA and PstC proteins. Two key residues (Gly-48 and Lys-49) have been shown to be required for phosphate transport by the Pst system (Cox et al., 1989) and are located in the conserved sequence associated with a nucleotide-binding site (Higgins et al., 1985). Importantly, these two residues are also conserved in the ORF74 and ORF75 products (Fig. 2).

Comparison of the organization of the pst operon of $B$. subtilis and that of $E$. coli is represented schematically in Fig. 3. It is very interesting that the gene corresponding to pho $U$, which is involved in the regulation of the phosphate regulon in E. coli (Wanner, 1987), is not present in the $B$. subtilis pst operon, while there are two genes homologous to $p s t B$ in $B$. subtilis. The location of $p h o U$ gene homologue in B. subtilis is not known at present.

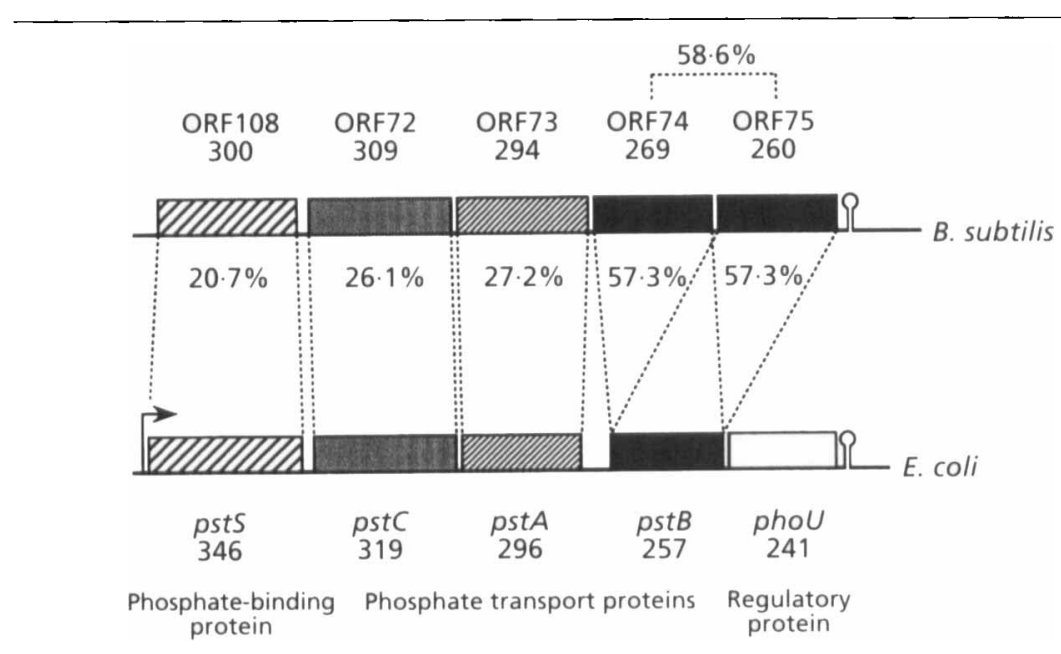

Fig. 3. Comparison of the organization of the pst operon of $B$. subtilis with that of $E$. coli. The percentage of identical amino acids between corresponding proteins is shown. Sizes of ORFs are to scale. Numbers below the gene name refer to the number of amino acid residues.

Fig. 1. Complete nucleotide sequence of the $B$. subtilis pst operon. Potential RBSs for the ORFs are underlined. Putative -35 and -10 promoter regions are doubly underlined. A potential rho-independent transcription terminator is indicated by arrows. The deduced amino acid sequences of the ORFs are shown in single-letter code with the respective termination codons indicated by asterisks. Two boxed sequences represent putative pho boxes. The consensus sequence for the pho box of $E$. coli consists of the $18 \mathrm{bp}$ sequence $5^{\prime}-C T(G / T) T C A T A(A T) A(A T) C T G T C A(C T)-3^{\prime}$ (Makino et al., 1986). All sequences were determined using the Taq Dye Primer Cycle Sequencing Kit and the 373A sequencer from Applied Biosystems as described previously (Takemaru et al., 1995). 


\section{ACKNOWLEDGEMENTS}

We are grateful to Dr Naotake Ogasawara for helpful comments and suggestions. We thank Dr Mitsuhiro Itaya for the kind gift of NotI linking clone, pNEXT27. This work was supported by a Grant-in-Aid for Creative Basic Research 'Human Genome Analysis' from the Ministry of Education, Science and Culture, Japan.

\section{REFERENCES}

Amemura, M., Makino, K., Shinagawa, H., Kobayashi, A. \& Nakata, A. (1985). Nucleotide sequence of the genes involved in phosphate transport and regulation of the phosphate regulon in Escherichia coli. J Mol Biol 184, 241-250.

Bachmann, B. J. (1990). Linkage map of Escherichia coli K-12, edition 8. Microbiol Rev 54, 130-197.

Cox, G. B., Webb, D. \& Rosenberg, H. (1989). Specific amino acid residues in both the $\mathrm{PstB}$ and $\mathrm{PstC}$ proteins are required for phosphate transport by the Escherichia coli Pst system. I Bacteriol 171, 1531-1534.

Gerdes, R. G. \& Rosenberg, H. (1974). The relationship between the phosphate-binding protein and a regulator gene product from Escherichia coli. Biochim Biophys Acta 351, 77-86.

Higgins, C. F., Hiles, I. D., Whalley, K. \& Jamieson, D. J. (1985).

Nucleotide binding by membrane components of bacterial periplasmic binding protein-dependent transport systems. EMBO J 4, 1033-1040.

Itaya, M. \& Tanaka, T. (1991). Complete physical map of the Bacillus subtilis 168 chromosome constructed by a gene-directed mutagenesis method. J Mol Biol 220, 631-648.
Makino, K., Shinagawa, H., Amemura, M. \& Nakata, A. (1986). Nucleotide sequence of the $p h o B$ gene, the positive regulatory gene for the phosphate regulon of Escherichia coli K-12. J Mol Biol 190, $37-44$.

Makino, K., Shinagawa, H., Amemura, M., Kimura, S., Nakata, A. \& Ishihama, A. (1988). Regulation of the phosphate regulon of Escherichia coli: activation of pstS transcription by PhoB protein in vitro. J Mol Biol 203, 85-95.

Ogasawara, N., Nakai, S. \& Yoshikawa, H. (1994). Systematic sequencing of the 180 kilobase region of the Bacillus subtilis chromosome containing the replication origin. DNA Res 1, 1-14.

Surin, B. P., Rosenberg, H. \& Cox, G. B. (1985). Phosphate-specific transport system of Escherichia coli: nucleotide sequence and gene-polypeptide relationships. J Bacteriol 161, 189-198.

Takemaru, K., Mizuno, M., Sato, T., Takeuchi, M. \& Kobayashi, Y. (1995). Complete nucleotide sequence of a skin element excised by DNA rearrangement during sporulation in Bacillus subtilis. Microbiology 141, 323-327.

Wanner, B. L. (1987). Phosphate regulation of gene expression in Escherichia coli. In Escherichia coli and Salmonella typhimurium: Cellular and Molecular Biology, vol. 2, pp. 1326-1333. Edited by F. C. Neidhardt, J. L. Ingraham, K. Brooks Low, B. Magasanik, M. Schaechter \& H. E. Umbarger. Washington, DC: American Society for Microbiology.

Wanner, B. L. \& Letterel, P. (1980). Mutants affecting alkaline phosphatase expression: evidence for multiple positive effectors of the phosphate regulon in Eschericbia coli. Genetics 96, 353-366.

Received 6 February 1996; revised 22 March 1996; accepted 29 March 1996. 